\title{
Overexpression of Liriodendron tulipifera JAG Gene (LtuJAG) Changes Leaf Shapes in Transgenic Arabidopsis thaliana
}

\author{
Lingmin Wei ${ }^{1}$, Shaoying Wen ${ }^{1}$, Zhonghua Tu ${ }^{1}$, Yanqing Zhao ${ }^{1}$ and Huogen $\mathrm{Li}^{1,2, * \mathbb{C}}$ \\ 1 Key Laboratory of Forest Genetics \& Biotechnology of Ministry of Education, Co-Innovation Center for \\ Sustainable Forestry in Southern China, Nanjing Forestry University, Nanjing 210037, China; \\ lmwei2019@njfu.edu.cn (L.W.); Winnie@njfu.edu.cn (S.W.); zhonghuatu@njfu.edu.cn (Z.T.); \\ zhaoyanqing@njfu.edu.cn (Y.Z.) \\ 2 College of Forestry, Nanjing Forestry University, Nanjing 210037, China \\ * Correspondence: hgli@njfu.edu.cn; Tel.: +86-025-8542-8731
}

Citation: Wei, L.; Wen, S.; Tu, Z.; Zhao, Y.; Li, H. Overexpression of Liriodendron tulipifera JAG Gene (LtuJAG) Changes Leaf Shapes in Transgenic Arabidopsis thaliana. Int. J. Mol. Sci. 2022, 23, 1322. https:// doi.org/10.3390/ijms23031322 Academic Editor: Pedro MartínezGómez

Received: 21 December 2021 Accepted: 20 January 2022 Published: 25 January 2022 Publisher's Note: MDPI stays neutral with regard to jurisdictional claims in published maps and institutional affiliations.

Copyright: (C) 2022 by the authors. Licensee MDPI, Basel, Switzerland. This article is an open access article distributed under the terms and conditions of the Creative Commons Attribution (CC BY) license (https:// creativecommons.org/licenses/by/ $4.0 /)$.

\begin{abstract}
In Arabidopsis thaliana, JAGGED (JAG) is a transcription inhibitor that controls the development of leaf polarity and regulates the expression of genes controlling lateral organ formation. Liriodendron tulipifera is an ornamental tree with extraordinary tulip-shaped flowers and goose weblike leaves, this is one of the suitable plants for morphological development research. To investigate the potential functions of the LtuJAG gene, we isolated the full-length LtuJAG from L. tulipifera, transferred it into $A$. thaliana via agrobacterium-mediated transformation, and monitored its expression pattern. Subcellular localization showed that LtuJAG was located in the nucleus. RT-qPCR assays indicated that $L t u J A G$ was expressed mainly in leaf buds and flowers, but not in mature leaves and stems. GUS staining results showed that $L t u J A G$ was expressed in the shoot apical meristem (SAM). Overexpressing LtuJAG changed A. thaliana leaf shapes, causing a moderate serration and a slight asymmetric distribution in the medio-lateral and proximal-distal axes. Ectopic expression of $L t u J A G$ induced the expression of lateral organ boundary suppressors JAGGED LATERAL ORGANS (JLO) and ARABIDOPSIS THALIANA HOMEOBOX1 (ATH1). It also repressed the expression of the apical meristem suppressor class-1 KNOX gene (KNOX $I$ ) and altered endogenous hormone levels. Our results suggest that $L t u J A G$ plays a role in negatively regulating leaf polarity formation in L. tulipifera.
\end{abstract}

Keywords: Liriodendron tulipifera; LtuJAG; leaf polarity; transformation; Arabidopsis thaliana

\section{Introduction}

The organ development of seed plants usually undergoes two opposite modes: (1) root and stem development, which displays overall radial symmetry; (2) development of lateral organs such as leaves and flowers, which show an obvious asymmetry [1]. The leaves of seed plants evolved from lateral branches, initiating from the peripheral zone (PZ) of the shoot apical meristem (SAM) and developing into flattened structures [2]. Genetic evidence has revealed that leaves underwent four morphological changes during their evolution: curling into a cone, developing short and flexible petioles as well as serrated edges, forming compound pinnate leaves, and stacking with each other along the branches to adjust the phyllotaxis and reduce drag [3]. Due to their morphological plasticity, leaves are ideal research subjects for under-standing organ morphogenesis and environmental adaptability.

The development of the leaves includes three processes: leaf initiation, leaf polarity establishment, and leaf morphology modulation [4]. Leaves emerge from a mass of stem cells at the SAM via lateral differentiation, thereby forming leaf primordia. The stem cells differentiate and proliferate along the asymmetric polarity of the adaxial-abaxial, medio-lateral, and proximal-distal axes to establish leaf polarity [5]. After the establishment of leaf polarity, its shape is formed by continues cell division and expansion. Many genes involved in leaf development have been identified, including KNOX I family genes, 
ASYMMETRIC LEAVES1 (AS1)/ROUGH SHEATH2 (RS2)/PHANTASTICA (ARP), PINFORMED1 (PIN1), BLADE-ON-PETIOLE 1/2 (BOP1/2), and CUP-SHAPED COTYLEDON (CUC) (Figure 1). The KNOX I family genes including SHOOT MERISTEMLESS (STM), BREVIPEDICELLUS (BP or KNAT1), KNOTTED IN ARABIDOPSIS THALIANA 2 (KNAT2), and KNAT6, which are essential for maintaining SAM activity, and their downregulation are required for leaf initiation [6-8]. This downregulation is contributed by ARP transcription factors. The AS1 and AS2 genes form a complex with the regulatory motifs CWGTTD (located on the BP promoter) and KMKTTGAHW (located on the KNAT2 promoters) [9]. Finally, they performed negative regulation of $B P$ and KNAT2 together [10]. BOP1 and $B O P 2$ can also repress $B P$ by directly promoting the expression of $A S 2$ [11,12] (Figure 1A). Moreover, $B O P 1$ and $B O P 2$ participate in the establishment of adaxial-abaxial polarity through $A S 2$ (adaxial fate regulator) activation and $Y A B B Y(Y A B$, abaxial fate regulator) repression [13] (Figure 1A). BOP1 and BOP2 proteins also function redundantly with AS1 and AS2 in the establishment of proximal-distal polarity [11] (Figure 1A). Notably, the PIN1-CUC regulation pathway plays important roles in leaf development-establishment of organ boundary and determining the location of serration during leaf morphology modulation [14] (Figure 1A,B). Moreover, SAWTOOTH 1 (SAW1) and SAW2 transcription factors also participate in leaf serration formation. The double mutations of $S A W 1$ and $S A W 2$ initiate the ectopic expression of $B P$ at the leaf margin, resulting in the lobed leaf and indicating that $S A W 1$ and $S A W 2$ are involved in the development of leaf margins by negatively regulating the expression of KNOX $I$ [15]. While the molecular development model from leaf primordia to maturity discussed above is generally accepted, the exact process of leaf morphogenesis is still unclear.

In recent years, some boundary genes have been found to be involved in the regulation of organ polarity. JLO has been shown to activate the expression of STM and BP and inhibit the expression of the auxin efflux transporter PIN1 [16], and JAG and its paralog $N U B B I N(N U B)$ are known to be negatively regulated by $B O P$ in lateral organs $[17,18]$ (Figure 1A). Further studies found that JAG positively regulates the expression of PIN1 in tomato leaves [19] (Figure 1B). Loss of function mutations at JAG sites leads to abnormal lateral organs in $A$. thaliana, including narrow leaves and serrated petals [20,21]. Research has also shown that $J A G$ controls the coordination of the cell cycle and growth in calyx primordia [22]. In addition, $J A G$ controls not only the growth rate of cells but also the polarized growth of organs. JAG and AS1/AS2 repress the expression of boundary-specific genes in sepals and petals to maintain normal organ initiation within the boundary zone [23]. Many studies have confirmed the regulatory role of $J A G$ in petal growth and development, but the molecular mechanism of JAG-mediated leaf morphogenesis is not clear. We set out to explore whether a better understanding of the expression pattern of LtuJAG may be helpful in improving the desired aesthetic qualities of leaves, thereby changing the utilization rate of light and other energy and assisting selective breeding programs for the improvement of ornamental tree species. 
A
$\rightarrow$ PIN1 polarity direction
$\rightarrow$ Positive intcraction
$\dashv$ Negative interaction
Auxin response maximum
CUC domain
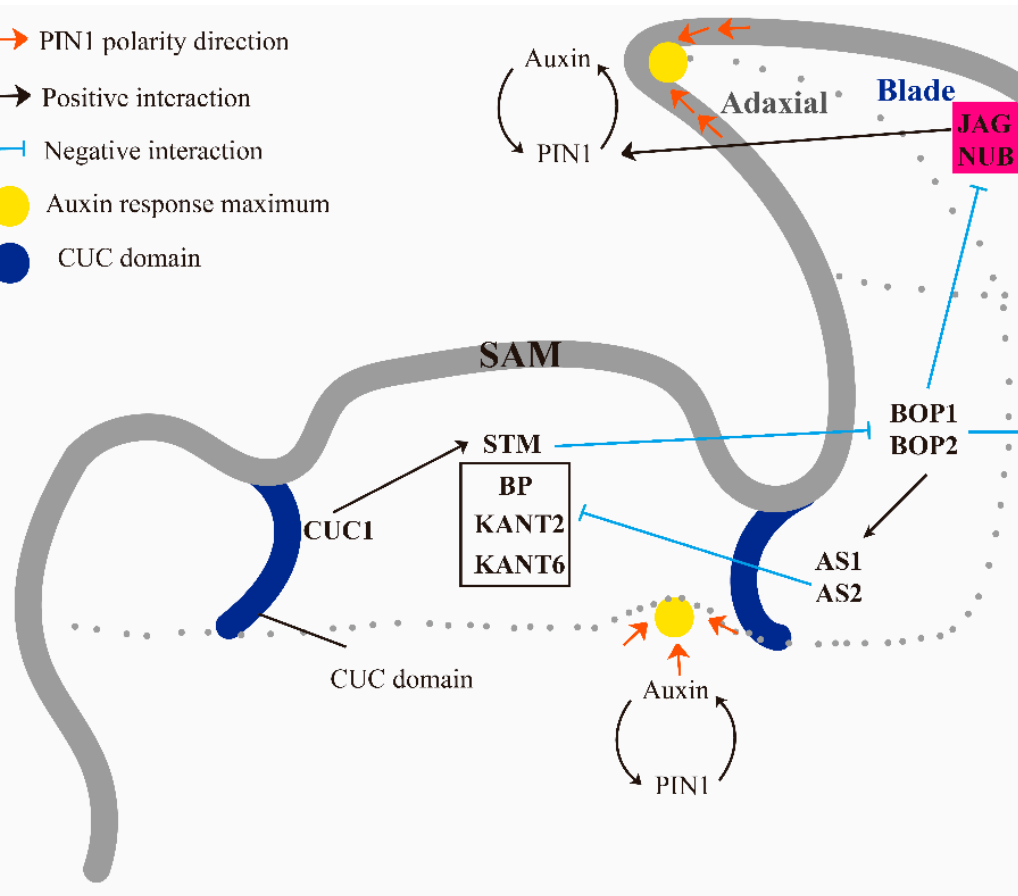

SAM

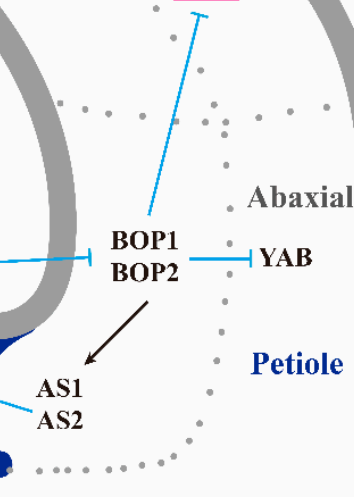

CUC domain<smiles>C1C[AlH2]CC[AlH2]CC[AlH2]1</smiles>

B

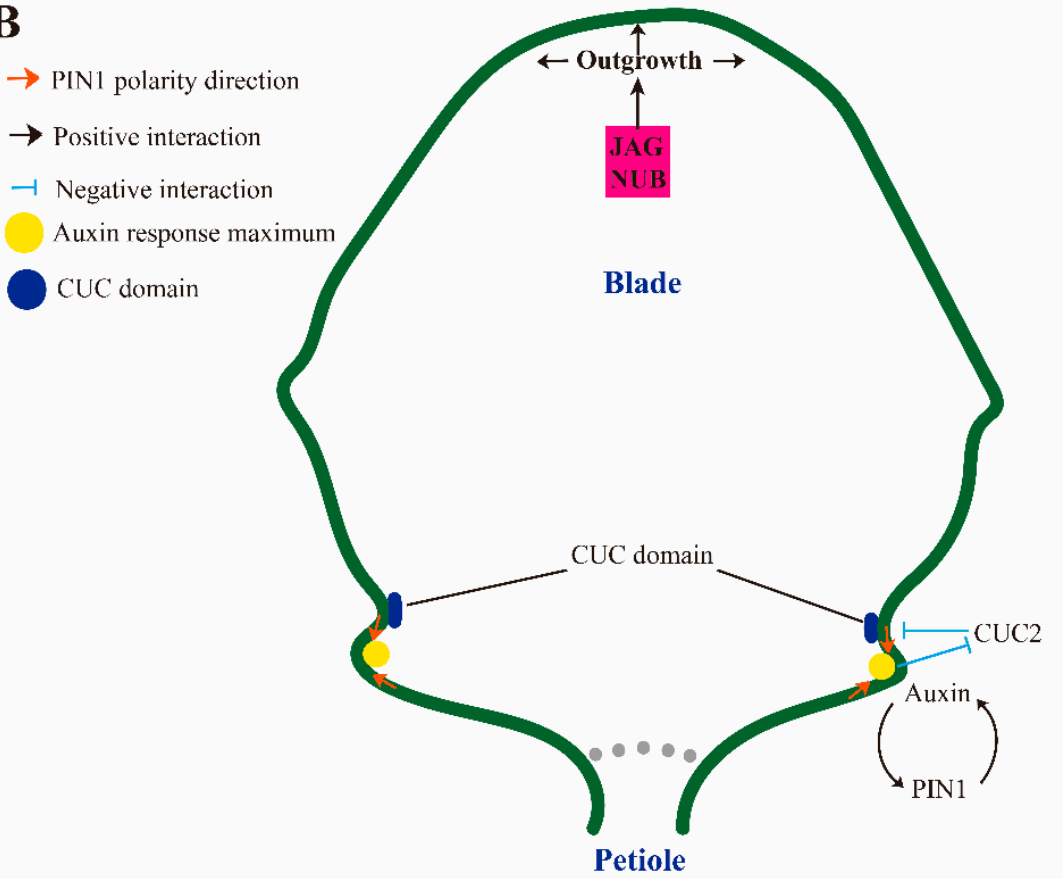

Figure 1. Regulation pattern of leaf margin morphogenesis in A. thaliana. (A) Regulation network controlling leaf polarity. $\mathrm{BOP}_{\mathrm{S}}$ in the petiole directly activate $A S 1 / 2$ to repress BP and exclude JAG-like factor from the petiole and restrict to the leaf margin thereby promoting leaf polarity differentiation; (B) Regulation network of leaf margin serration induced by boundary gene. JAG promotes PIN1 convergence at the tip of serrations along leaf margin and interacts with boundary genes CUC2.

\section{Results}

\subsection{Cloning and Sequence Analysis of LtuJAG}

Full-length LtuJAG (1344 bp; ORF, 801 bp) encoded a protein comprising 267 amino acid residues with a molecular weight of $29.50 \mathrm{kDa}$ and a theoretical isoelectric point $(\mathrm{pI})$ of 8.05. The hydrophilicity of the protein was -0.856 with an instability coefficient of 58.05 , 
indicating that it was likely to be unstable and hydrophobic. The amino acid sequence of LtuJAG was used as a query to perform a BLASTP search to explore its structural conservation and phylogenetic position. Thirteen homologous protein sequences from other plant species showed that the $N$-terminus comprising the $\mathrm{C}_{2} \mathrm{H}_{2}$ zinc-finger domain was conserved while the EAR motif was not, indicating that $L t u J A G$ belongs to the $\mathrm{C}_{2} \mathrm{H}_{2}$ zinc-finger family (Figure 2A). The alignment of these sequences showed a high degree of conservation relative to LtuJAG, varying from 91.35\% (Magnolia wufengensis, QAY29215.1) to 51.05\% (Populus euphratica, XP_011007835.1). The phylogenetic tree showed that JAG proteins from L. tulipifera and M. wufengensis were clustered together (Figure 2B).
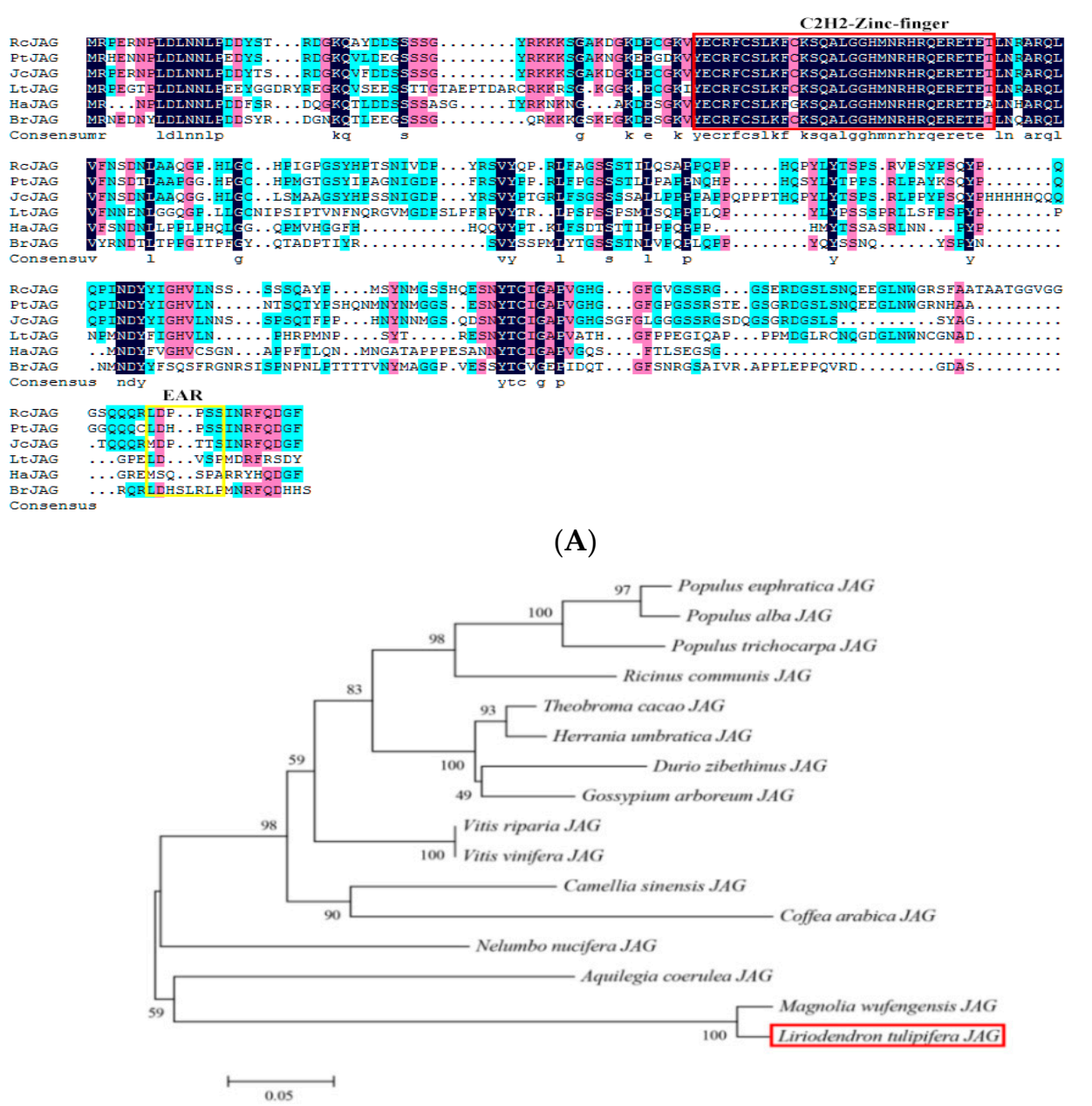

(B)

Figure 2. Sequence and phylogenetic analyses of LtuJAG protein. (A) The multiple sequence alignment of LtJAG protein with JAG-like protein in Ricinus communis (RcJAG, XP_025015302.1); Populus trichocarpa (PtJAG, XP_024466075.1); Jatropha curcas (JcJAG, XP_020538088.1); Helianthus annuus (HaJAG, XP_021975637.1); Brassica rapa (BrJAG, XP_009105303.1). The highly conserved core sequence Homeodomain is represented by a red box. The EAR motif is indicated by a yellow box; (B) Phylogenetic relationships among JAG proteins from different plant species. LtuJAG is indicated by the red box.

\subsection{Subcellular Localization of LtuJAG}

Subcellular localization using LOCALIZER (http:/ / localizer.csiro.au/) (accessed on 2 July 2020) [24] predicted that LtuJAG would be found in the nucleus. Green fluorescent protein (GFP) signals of the 35S::eGFP construct were inserted into the nucleus and cell membrane, while the signal of LtuJAG::eGFP was only localized in the nucleus of $N$. benthamiana leaves (Figure 3B). DAPI $\left(4^{\prime}, 6^{\prime}\right.$-diamidino-2-phenylindole) dye was utilized 
to stain the nucleus to stimulate a fluorescent blue signal. The resulting fluorescent cyan signal further confirmed that LtuJAG was located in the nucleus, which is consistent with transcription factor patterns (Figure 3B).

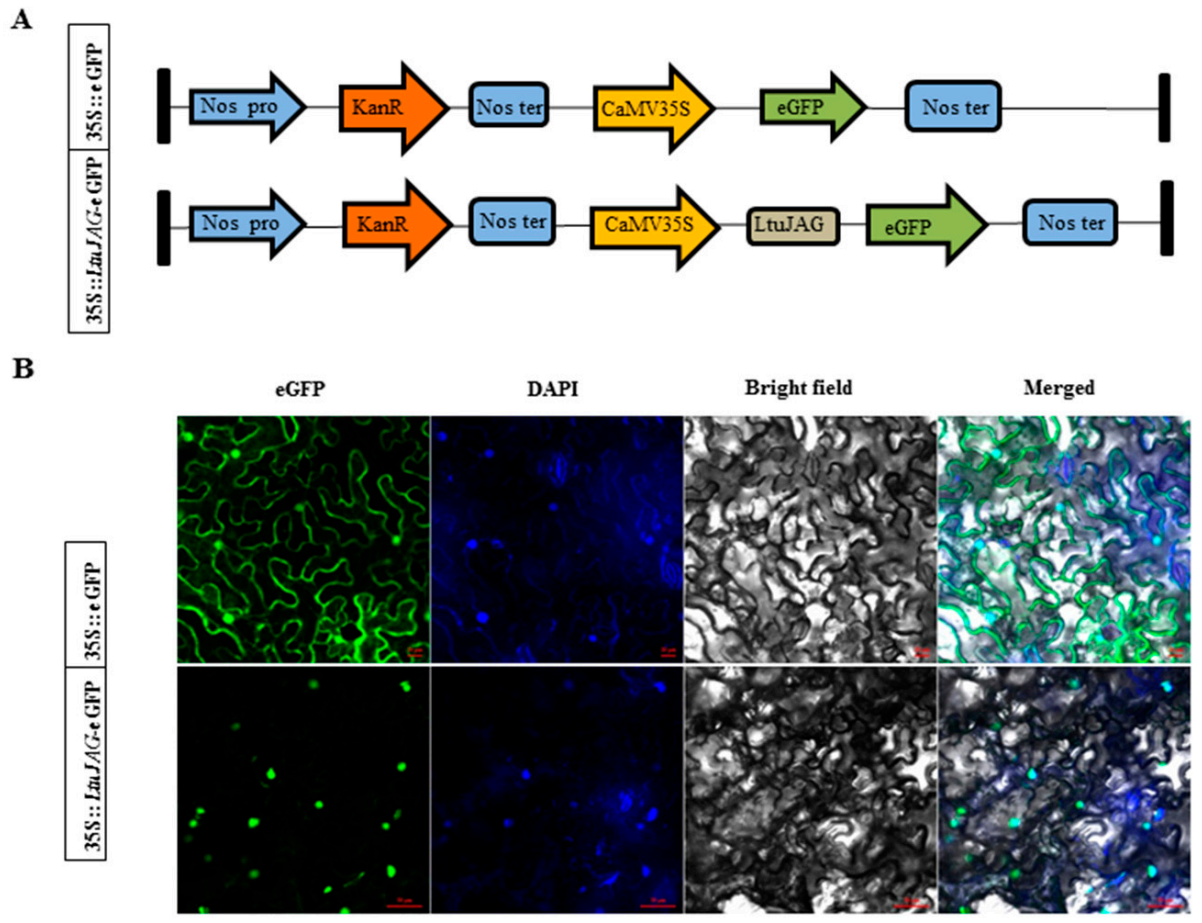

Figure 3. Subcellular localization of LtuJAG protein. (A) The construct of 35S::LtuJAG-eGFP and 35S::eGFP (eGFP: green fluorescent protein; NOS: nopaline synthase gene; KanR: Kanamycin resistance gene); (B) Subcellular localization of the 35S::LtuJAG-eGFP protein in tobacco epidermal cells. At 2 days after transfection, eGFP and DAPI ( $4^{\prime}, 6^{\prime}$-diamidino-2-phenylindole, and nucleus specific dye) signal was observed by confocal fluorescence microscopy, 35S::eGFP was used as a control. Scale bars $=20$ and $50 \mu \mathrm{m}$.

\subsection{Tissue Expression Pattern of LtuJAG}

To verify the expression pattern of LtuJAG in different tissues of L. tulipifera, we examined the mRNA of LtuJAG in eight L. tulipifera tissues (Figure 4A). Expression in flower buds was significantly higher than that of the other tissues, followed by expression in the stamen, petals, calyx and leaf buds, with stems and leaves showing the not expression. This suggests $L t u J A G$ may play an important role in the development of flower organs.

The time-specific expression pattern of $L t u J A G$ during leaf development determined by sampling the leaf bud growth stage (stages 1 and 2), young leaf stage (stage 3), mature stage (stages 4 to 6), and senescence stage (stage 7) showed that LtuJAG expression increased with the gradual expansion of leaf buds at the bud growth stage (Figure 4B). The expression of $L t u J A G$ decreased with the growth of leaves at the leaf-spreading stage. LtuJAG was not expressed during leaf maturation and senescence, indicating that it may be expressed during vigorous meristem growth of L. tulipifera but does not participate in the regulation of leaf growth and development at maturity.

To further confirm the leaf space-specific expression pattern of LtuJAG, we divided the leaf margin of L. tulipifera into tooth tips and sinuses. LtuJAG expression was markedly higher in the petiole of the leaf than in the other parts, and it was generally higher in leaf tooth sinuses (d) than in leaf tooth tips (a, c, and e) (Figure $4 \mathrm{C}$ ). These results suggest that $L t u J A G$ may play a direct regulatory role in leaf margin development as well. 
A

$\mathbf{C}$
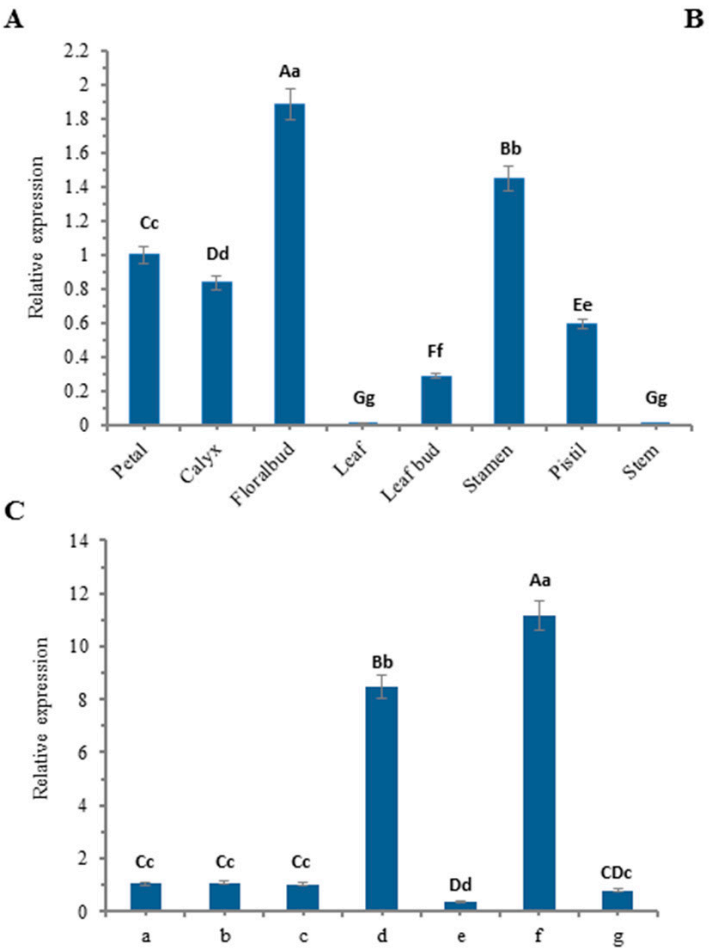

B

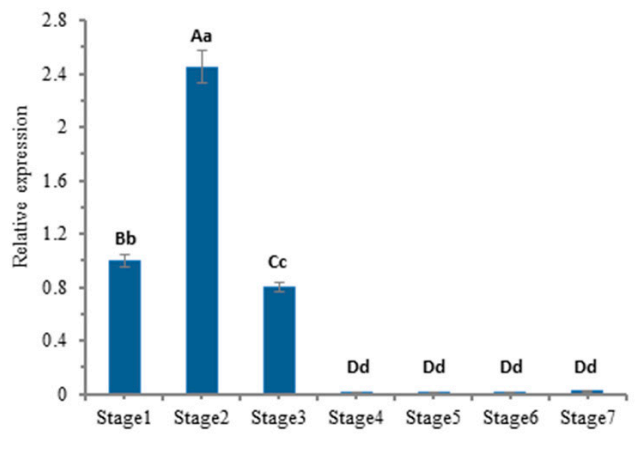

Figure 4. Quantitative RT-PCR analysis of transcript levels of LtuJAG in L. tulipifera. LcACTIN97 gene was used as an internal control to normalize the values of target transcripts. (A) Transcript levels of LtuJAG in different tissues; (B) Transcript levels of LtuJAG at different stages of leaf development; Stage 1-2: leaf bud growth stage; Stage 3: young leaf stage; Stage 4-6: mature stage; Stage 7: senescence stage; (C) Transcript levels of LtuJAG at different parts of leaf in L. tulipifera, a, $\mathrm{c}$ and e: leaf tooth tip, $\mathrm{b}$ and $\mathrm{d}$ : leaf tooth sinus, g: middle of leaf, f: petiole. Date represented the means $( \pm \mathrm{SD})$ from the three replicates, and the error bars represent the standard deviation between the replicates. Data were analyzed statistically using the one-way ANOVA Tukey's test. Different letters indicate significant differences at $p<0.01$ and $p<0.05$ level.

\subsection{LtuJAG Promoter Is Leaf-Specific in Transgenic A. thaliana}

To detect tissue specificity, the $L t u J A G$ promoter fragment (named $p L t u J A G$ ) located approximately $2 \mathrm{~Kb}$ upstream of the JAG gene in L. tulipifera, was cloned, sequenced and analyzed for potential regulatory elements (Table S2), including light-responsive cis-elements, such as Box 4, G-box and I-box, as well as TCCC-motifs. The abiotic stress response elements included LTR (low temperature), MYC (cold), STRE (thermal induction), TCrich repeats (defense and stress responsiveness), and WRE3 (damage). Furthermore, a few hormone-responsive elements were identified, such as ABRE (ABA), AuxRE (Auxin), AuxRR-core (Auxin), ERE (ethylene), TATC-box (GA), and TCA-element (salicylic acid). Sequence analysis of the LtuJAG promoter region implies that the LtuJAG gene may be implicated in regulating a range of processes, including responses to light signals, abiotic stresses, and hormones.

To confirm the core elements responsible for meristem development, the $p L t u J A G$ sequence was fused to the GUS reporter gene (pLtuJAG::GUS) and transformed into A. thaliana, pLtuJAG-induced GUS expression was observed during the two-leaf, four-leaf, six-leaf and flowering stages of $\mathrm{T}_{2}$ overexpression lines using histochemical staining (Figure 5A,B). After germination for 2 days, GUS activity of seeds and seedlings was not expressed. At the stage of leaf bud differentiation (4-12 days), GUS expression was not detected in the radicle. Concurrently, the $L t u J A G$ promoter drove GUS to be stably expressed in leaves and hypocotyls. With the growth of seedlings, GUS activity in leaf buds gradually decreased, while gradually increasing in newly differentiated leaves (Figure 5B). It was only expressed in leaves, but not in inflorescences, pods, and stems at the repro- 
ductive stage (Figure 5B). Furthermore, we observed that the promoter activity of LtuJAG was the strongest in the main leaf vein, followed by the secondary leaf vein, and weak GUS expression was detected in mesophyll cells, indicating that the LtuJAG promoter is exclusively expressed in leaf organs. From these observations, it can be inferred that pLtuJAG is a leaf bud meristem-specific promoter.

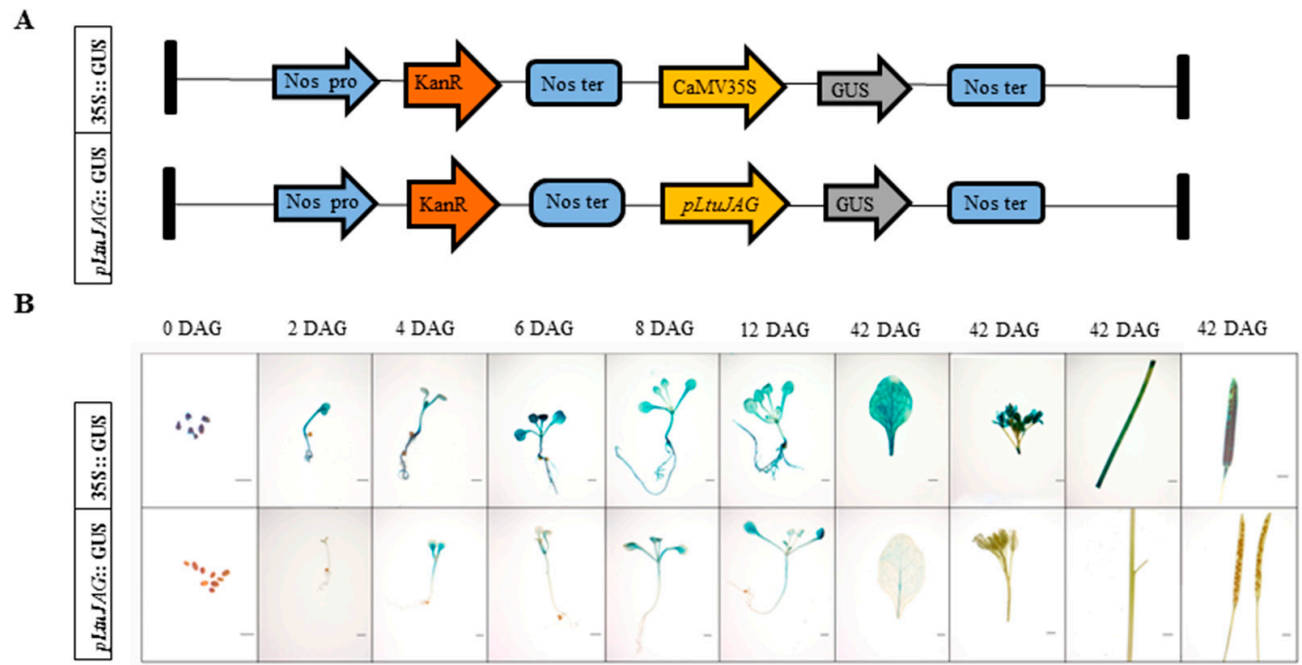

Figure 5. GUS histochemical assays in transgenic Arabidopsis $\mathrm{T}_{2}$ overexpression lines. (A) The T-DNA construction diagram used for Arabidopsis transformation. GUS: $\beta$-glucuronidase gene; Nos: nopaline synthase gene; KanR: Kanamycin resistance gene; (B) Histochemical staining in seedlings (two-leaf stage, four-leaf stage and six-leaf stage), rosette leaf, stem, pods, inflorescences, and seeds sampled during the vegetative and reproductive stage form transgenic $\mathrm{T}_{2}$ Arabidopsis seedlings harboring constructs with GUS expression driven by the CaMV 35S promoter (35S::GUS) and $p$ LtuJAG. Photographs were taken 2 days, 4 days, 6 days, 8 days, 12 days, and 42 days after seed germination. DAG: day after germination. Scale bar $=1 \mathrm{~mm}$.

\subsection{Phenotype of Transgenic A. thaliana}

The coding sequence of $L t u J A G$ was fused to the 35 S promoter (35S::LtuJAG) and transferred into $A$. thaliana. Approximately $32 \mathrm{~T}_{1}$ generation positive plants were produced. Seven of these overexpression lines had the phenotype of small leaf area (LtuJAGOE11), including two with a phenotype producing serration, LtuJAG-OE7 and LtuJAG-OE8 (Figure 6A,C). The transgenic line 35S::LtuJAG-OE7 exhibited a phenotype causing small leaf area, we also measured lamina length, width, and area in wild type and overexpression lines. Compared with the wild type, the petiole of the overexpression strain became longer (Figure 6E), the lamina length, width, and area were reduced (Figure 6F,G,I) The ratio of lamina length to width remained unchanged (Figure 6I), indicating that the overexpression line 35S::LtuJAG-OE7 altered leaf size by simultaneously reducing the rate of leaf cell expansion in both the medio-lateral and proximal-distal axis directions. A range of defects was observed, including irregular curls and lobes at the distal ends of the leaves (Figure 6D), thinner mesophyll tissue in the lobed part of the leaves, abnormal vein growth, and narrowing of the proximal ends forming a wedge. This latter also induced some petiole elongation, and only petioles remained in some severely defective leaves. This phenotypic change in the absence of leaf cells suggests suppression of leaf polarity had occurred.

The transgenic line 35S::LtuJAG-OE8 exhibited a phenotype causing petiole elongation and leaf serration at the four-leaf stage (Figure 6B) while showing dorsal leaf margin curling at the mature stage (Figure 6A,C). This was caused by accelerated proliferation and expansion of mesophyll cells on the adaxial blade, while the cell division speed on the abaxial blade remained unchanged. To a greater extent, the leaves showed a moderate serration and slight asymmetric distribution along the medio-lateral axis, which was consistent with the heteromorphic leaves of a mutant [20]. 
A

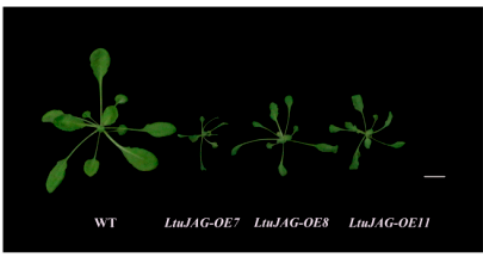

C

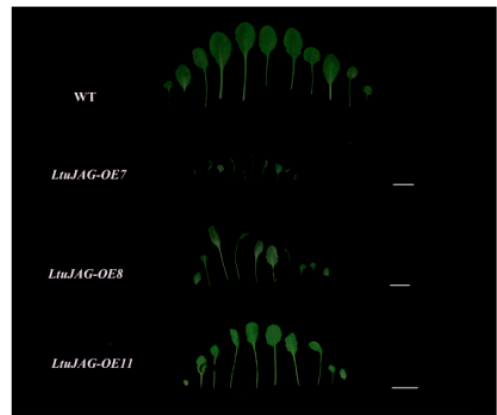

E

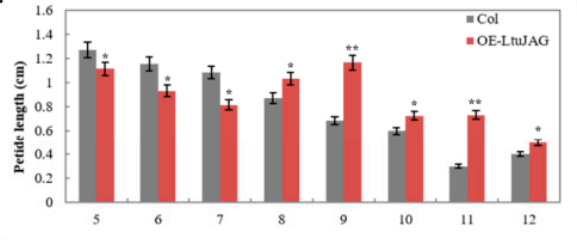

F
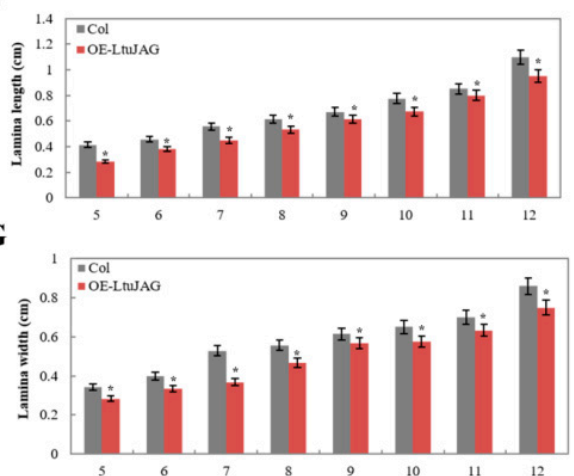

H

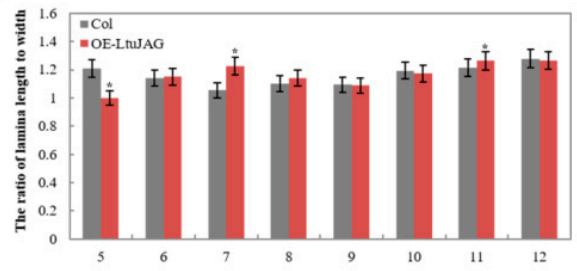

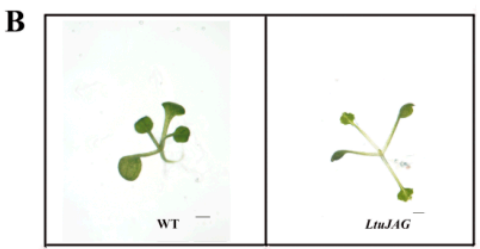

D

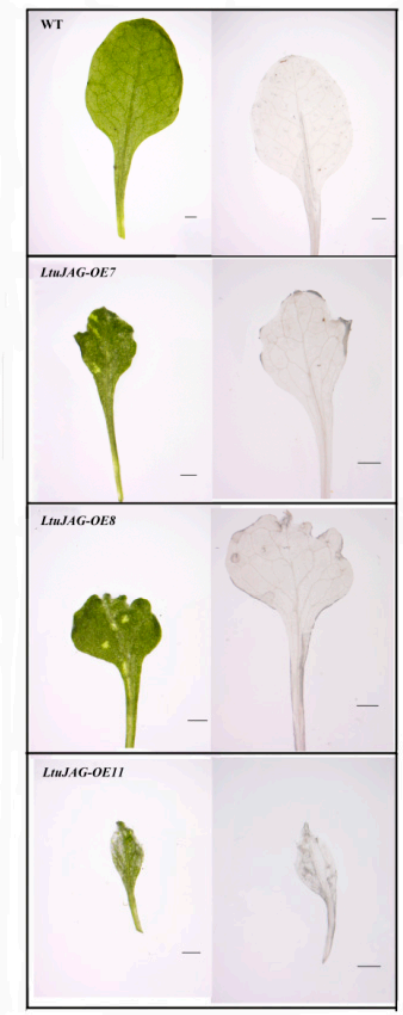

I

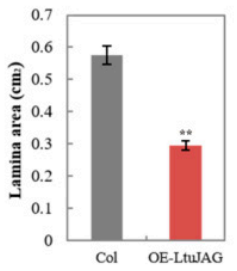

Figure 6. The margin serration phenotype of transgenic LtuJAG lines. (A-D): The transgenic line 35S::LtuJAG exhibited a phenotype causing petiole elongation, leaf serration and small leaf area at the 10 days $(\mathbf{B})$ and 32 days $(\mathbf{A}, \mathbf{C}, \mathbf{D})$ after seed germination. Scale bar $=1 \mathrm{~mm}$ and $1 \mathrm{~cm}$; $(\mathbf{H}, \mathbf{I})$ : Quantification of leaf lobes of transgenic LtuJAG lines, Col-0 was used as a wild-type control; (E): Quantification of the petiole lengths of 5th leaves to 12th leaves; (F): Quantification of the lamina length of 5th leaves to 12th leaves; (G): Quantification of the lamina width of 5th leaves to 12th leaves; (H): Quantification of the ratio of the lamina length to width; (I): Quantification of lamina area of 5 th leaves. Leaves of plants grown for 32 days were examined, error bars showed SD $(n \geq 12)$. The asterisks indicate significant difference from the WT using the Student's $t$-test $\left({ }^{* *} p<0.01,{ }^{*} p<0.05\right)$. 


\subsection{Expression of Genes Related to Leaf Development in Transgenic A. thaliana}

Since the formation of leaf morphology occurs during the transition from leaf primordia initiation to polarity establishment, we selected 22 genes that have been reported to play a part in this process. The heterologous overexpression of $L t u J A G$ resulted in a significant increase in the expression of LtuJAG in overexpression lines (OE7, OE8, and OE11), indicating that we successfully identified the overexpression lines of $\mathrm{T}_{3}$ generation (Figure 7 ). Compared with the wild type, 35S::LtuJAG increased the expression of IPT7 and decreased the expression of GA20ox1 at the leaf primordia, suggesting that JAG promotes antagonism between GA20ox1 and IPT7, thus maintaining the dynamic balance of hormones during development. 35S::LtuJAG downregulated the expression of STM, KNAT2, SBP-LIKE9 (SPL9), and CUC2/3, and upregulated the expression of AS2, JLO, BOP1, SAW1/2, ATH1, AUX1 and TEOSINETE BRANCHED1/CYCLOIDEA/PROLIFERATING CELL FACTOR 4 (TCP4) at the leaf primordia. The expressions of KNAT1, KNAT6, PIN1, AS1, BOP2, TCP2, and $E R$ were not significantly different between WT and the overexpression lines (Figure 7). Therefore, we speculate that JAG may participate in leaf primordia differentiation and leaf polarity formation through multiple regulatory pathways, but determining by which regulatory pathways would require further investigation.

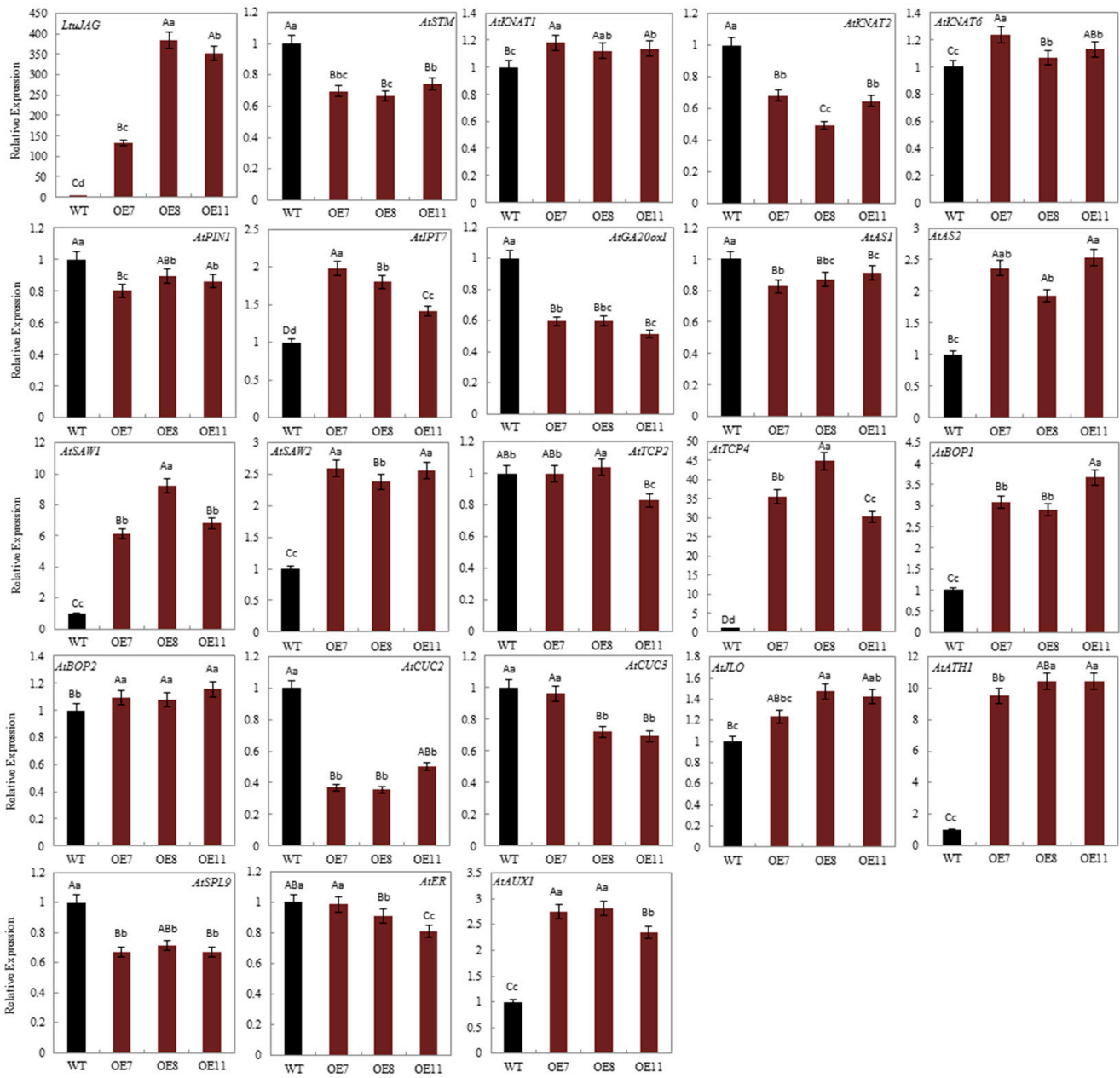

Figure 7. Quantitative RT-PCR analysis of $L t u J A G$ and genes in relation to leaf development in the ten-day-old wild type (WT) and three independent LtuJAG overexpression lines, OE7, OE8 and OE11. ACTIN2 served as the reference gene. The value of genes expression level in WT was set to "1" as a control, the error bars represent the standard deviation among three biological replicates. Data were analyzed statistically using the one-way ANOVA Tukey's test. Different letters indicate significant differences at $p<0.01$ and $p<0.05$ level. 


\section{Discussion}

\subsection{Domain Function in LtuJAG}

A 1344 bp cDNA full-length sequence named LtuJAG encoding 267 amino acid proteins was identified within the leaf bud cDNA of L. tulipifera. Sequence analysis of Ltu$J A G$ showed that $L t u J A G$ had similar conserved domains with JAG in Ricinus communis, Populus trichocarpa, Jatropha curcas, Helianthus annuus, and Brassica rapa. A single 31 amino acid $\mathrm{C}_{2} \mathrm{H}_{2}$ type Zinc- finger motif (amino acid position 60-91), which may have a role in DNA binding, and a short nine amino acid ERF-associated amphiphilic expression (EAR) motif (amino acid positions 250-258) at the amino acid end were observed to function as repressors. These results demonstrate that JAG belongs to the $\mathrm{C}_{2} \mathrm{H}_{2}$ type zinc-finger transcription factor family. Homology analysis showed that the amino acid sequence of LtuJAG was $51.05-91.35 \%$ similar to that of other species, especially the woody plant $M$. wufengensis. LtuJAG is predicted to function similarly to $A t J A G$, a class of transcriptional inhibitors that regulate processes involved in organ morphological development and polar growth [25].

Promoters are central to processes of transcriptional regulation and fundamental to the study of gene expression and regulation. Analysis of cis-acting elements showed that their most common products were hormones, such as aux (AuxRE), GA (TATC-box), abscisic acid (ABRE), salicylic acid (TCA-element), and ethylene (ERE). The functions of auxin and GA are associated with the developmental function of $J A G$ in regulating the three-dimensional morphology of plant organs. Moreover, plants synthesize abscisic acid to initiate defense mechanisms against stress [26]. Salicylic acid and ethylene are endogenous signaling molecules that activate plant hypersensitivity and systemic acquired resistance $[27,28]$. MYB and WRKY are involved in regulating the dual response of plants to biotic and abiotic stresses $[29,30]$. Therefore, we speculate that the LtuJAG gene is a transcription factor that participates in regulating development and resistance to stress.

\subsection{Expression Pattern of LtuJAG during Leaf Shape Formation}

Our study showed that LtuJAG was located in the nucleus of tobacco, while by observing AtJAG::GFP fusion protein under control of AP1 promoter in the epidermal cells of pedicels of flower primordia, AtJAG was located in the nucleus. This suggests that JAG has the characteristics of a typical transcription factor [21]. LtuJAG was specifically expressed in flower organs, such as flower buds, petals, and stamens. High concentrations of $J A G$ RNA in rice have been detected in the inflorescence meristem, axial meristem, flower organ primordia, stamen, pistil, anther, and integument [31-33], demonstrating that $J A G$ has a certain impact on the development of each floral organ. $L t u J A G$ was only expressed in leaf buds at the vegetative stage, and not in mature leaves or stems, which is consistent with other observations of $A$. thaliana by Dinneny et al. [21]. To gain further insight into the expression pattern of $L t u J A G$, we observed that the $L t u J A G$ promoter was stably expressed in leaves and hypocotyls at the vegetative stage of Arabidopsis, and its expression in the leaves gradually increased over time. AtJAG promoter-driven GUS gene expression was highest in the radicles and hypocotyls $12 \mathrm{~h}$ after germination, gradually decreased in the cotyledons and hypocotyls at 3 and 4 days, and increased in the radicles [34], which further explains the specific expression of $J A G$ at the early stage of apical meristem differentiation. In conclusion, we demonstrated that $J A G$ is a transcription factor that regulates leaf and floral organ development.

\subsection{Overexpression of LtuJAG Results in Leaf Polarity Defect}

The adaxial axes of leaf primordia face towards the meristem while abaxial axes face away from it. The formation of the adaxial-abaxial axis is fundamental for establishing the medio-lateral axis and proximal-distal axis of the leaf [1]. Overexpression of LtuJAG in A. thaliana resulted in the curling of adaxial tissue towards the abaxial axis. Cytological observations indicate that defects within the thick-walled cells on the abaxial axis of leaves are responsible for curling [35], indicating that $L t u J A G$ affects cell development. Intrigu- 
ingly, we found that $L t u J A G$ was ectopically expressed in leaves with deep serrations at the tip and margin. Study of KIP-RELATED PROTEIN2 (KRP2) revealed that the appearance of deep serration was caused by a proliferation defect in total cells, and the degree of lobe formation was related to the number of cells [36]. Moreover, the SHALLOT-LIKE1 (SLL1) gene in rice controls the development of leaf adaxial thick-walled cells by regulating programmed cell death (PCD) to establish leaf adaxial polarity [35]. Thus, the rate of cell division and proliferation can directly affect the final morphology of an organ, but the mechanisms by which cell division is controlled are not well understood. On the other hand, the establishment of adaxial-abaxial, proximal-distal, and medio-lateral axis polarities appears to be coordinated rather than independent of each other. How the development of these three polarities is coordinated remains to be discovered.

\subsection{LtuJAG Overexpression Alters the Expression of Genes Related to Leaf Morphogenesis}

Numerous studies have confirmed the inhibitory effect of $J A G$ on the development of petal polarity, and that petals are derivatives of the apical meristem and homologues of leaves [20-23]. Therefore, we speculated that JAG has an effect on the development of leaf polarity. The mutation of $J A G$ in Arabidopsis resulted in edge serration of leaves and petals [21]. The overexpression of $J A G$ promoted the fusion of cotyledons to varying degrees and had no effect on the growth of mature leaves [20]. We confirmed that LtuJAG influenced the establishment of three polar axes of leaves, and further examined the expression of related genes. We observed that $L t u J A G$ significantly affected other developmental genes at the differentiation stage of leaf primordia. We further confirmed that LtuJAG significantly downregulated the expression of the KNOX I gene, STM, and KNAT2, but had little effect on KNAT1 and KNAT6. This expression pattern is also consistent with the finding that KNAT1 and KNAT6 do not participate in the regulation of early morphogenesis in leaf primordia $[7,8]$. Previous studies have confirmed that $A S 1 / 2, B O P 1 / 2$, and $S A W 1 / 2$ act as negative regulators of KNOX $I$ in the development of leaf primordia $[9,11]$. Our study found that $L t u J A G$ can upregulate the expression of the KNOX I negative regulator, indicating that JAG may indirectly downregulate the expression of KNOX I during the development of lateral meristematic tissue. LtuJAG also accelerated the expression of the meristem boundary inhibitor genes ATH1 and JLO. ATH1 induces the expression of KNOX I downstream genes by forming heterodimers with STM and BP [37]. Similarly, JLO can activate the expression of STM and BP [16]. In conclusion, we speculate that JAG may synergize or promote $K N O X I$ upstream activators in leaf primordia differentiation and participate in the positive regulation of KNOX I upstream inhibitors.

Other boundary inhibitors, such as $C U C 1 / 2 / 3$, have been found to be activated by STM during the development of apical meristem, and CUC2 is regulated by STM and restricted to the SAM boundary region [38]. The inactivation of TCP function contributes to the excessive proliferation of leaf margins, resulting in leaf expansion and curled or serrated leaflets [39-41]. Regulation of leaf margin development by TCP transcription factors is accomplished by the negative regulation of CUC1/2/3. TCP4 forms dimers with CUC2 and CUC3, respectively, inhibiting the expression of CUC2/3 [42]. SPL9 competitively binds TCP to form dimers with CUC [42], and it is likely that the expression of SPL9 also affects the expression of CUC. This study found that LtuJAG can increase the expression of TCP 4 and downregulate the expression of SPL9 and CUC2/3, indicating that $J A G$ can also participate in the TCP4-CUC2/3 regulation pathway, but whether it is relative by JAG regulating SPL9 expression with this pathway requires further investigation.

Gibberellin and brassinolide control leaf growth by promoting cell proliferation and expansion $[43,44]$. PIN1 is also required for organ initiation, boundary restriction, and growth [45]. ERECTA (ER) can control cell proliferation by inhibiting auxin expression [46]. In LtuJAG overexpression lines, the expression of PIN1 and ER was not significantly different from that of the wild type, indicating that JAG may not be directly related to auxin biosynthesis. As a downstream target gene of KNOX I, IPT7 in A. thaliana was induced to be expressed by STM during apical meristem differentiation [47]. STM also 
inhibited gibberellin activity by reducing the level of GA20ox1. In addition, the expression of GA20ox1 in tomato was negatively correlated with the degree of leaf margin serration, whereas IPT7 was positively correlated with it [48]. LtuJAG significantly upregulated the expression of IPT7 and decreased the expression of GA20ox1, indicating that JAG can also participate in the antagonism between hormones, thereby contributing to the differentiation of meristem organs. Whether it regulates hormones through the KNOX I pathway requires further research. In conclusion, we speculate that the boundary limiting factors JLO and $A T H 1$, the upstream activators of the KNOX I gene, were directly activated by JAG, resulting in leaf primordia with disorganized polarity that sometimes fail to complete morphological development(Figure 8), but further studies are needed to determine the validity of this hypothesis. The mechanism by which genes controlling leaf morphogenesis interact at different developmental stages is unclear. Investigating this will be a focus of our future research.

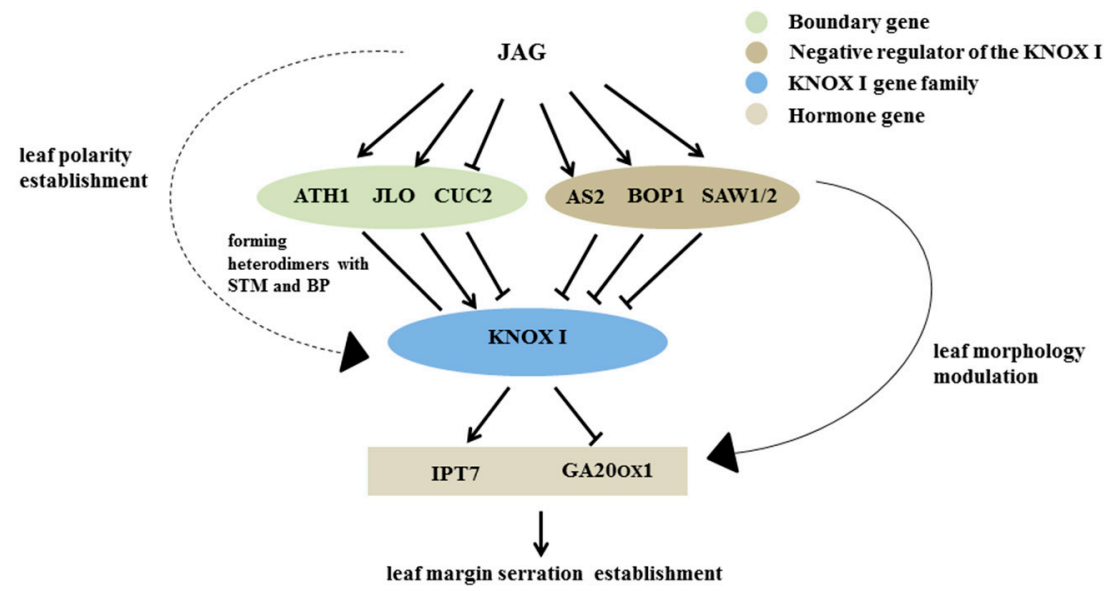

Figure 8. Proposed model for the regulatory mechanism of $J A G$. KNOX I family members are differentially expressed during different developmental stages of plants. The initiation and polarity establishment of leaf organs need to trigger genes like $A S 1 / 2, B O P 1 / 2, S A W 1 / 2$ to inhibit the expression of KNOX I (STM and BP). During the development of leaf margin serration, KNOX I genes (STM, KNAT2 and KNAT6) positively regulate the serration degree. JLO induces and activates the expression of STM and BP. ATH1 forms heterodimer with STM and BP.While CUC2 can significantly inhibit the expression of KNAT2 and KNAT6 genes. JAG promotes the expression of JLO (the activating gene of KNOX I), and inhibits the expression of CUC2 (the inhibitor of KNOX I) thereby promoting the expression of KNOX I through two-way regulation. KNOX I (STM and BP) further promotes the expression of cytokinin gene IPT7 and inhibits the expression of gibberellin synthesis gene GA20ox1, thereby regulating the hormone level at the leaf margin and finally establishing the leaf serration morphology. The solid arrows indicate results that have been experimentally determined, whereas dashed arrows indicate speculated effects supported by the literature.

\section{Materials and Methods}

\subsection{Plant Materials and Growth Conditions}

We found that the number of leaf serration increased with time in L. tulipifera and ended at senescence. Moreover, in a previous study, we had determined the timing and location of leaf initiation, leaf polarity establishment, leaf serration formation and leaf morphogenesis in L.chinense and L. tulipifera using SEM and paraffin section observation [49]. Our focus was on whether LtuJAG genes were involved in the process of altering the number of leaf serrations. Therefore, L. tulipifera trees were studied, originating from South Carolina, USA, were planted in a provenance trial plantation in the Xiashu Forest Farm in Jurong County, Jiangsu Province, China $\left(119^{\circ} 13^{\prime} 20^{\prime \prime}\right.$ E, $\left.32^{\circ} 7^{\prime} 8^{\prime \prime} \mathrm{N}\right)$ in 1993 [50].

To investigate the expression pattern of LtuJAG in different tissues, leaves, stems, stamens, pistils, flower buds, sepals, leaf buds, and petals of L. tulipifera were collected in April 2018. To verify the time-specific expression pattern, leaves at different stages of 
development (leaf bud germination, young leaves, mature leaves, and senescence) were collected from March to August 2018 (Figure 9A). As our focus lies in studying the leaf polarity establishment, leaf serration formation and leaf morphogenesis, we collected leaf buds and leaf from the corresponding stages, namely leaf bud germination stage (P1 and $\mathrm{P} 2$ ), young leaf stage (P3), leaves with three lobes, five lobes and six lobes, divided into mature stage (P4 and P6) and senescence stage(P7). Current studies emphasized that the generation of leaf margin serrations requires hormonal convergence at the leaf tooth tip, while boundary genes are confined at the leaf tooth sinus [45]. To explore the effect of $J A G$ genes as boundary suppressors on different parts of leaf in L. tulipifera, leaves were categorized into the protruding parts of the leaf margin ( $a, c$, and e), concave parts of the leaf margin (b and d), petiole (f), and the middle part of the leaf (g) (Figure 9B), three replicates from each of the seven samples (approximately $1 \mathrm{~g}$ each) were separated using sterilized scissors. All samples were frozen immediately in liquid nitrogen and stored at $-80{ }^{\circ} \mathrm{C}$ for RNA extraction, subsequent molecular cloning and real-time quantitative PCR.

A

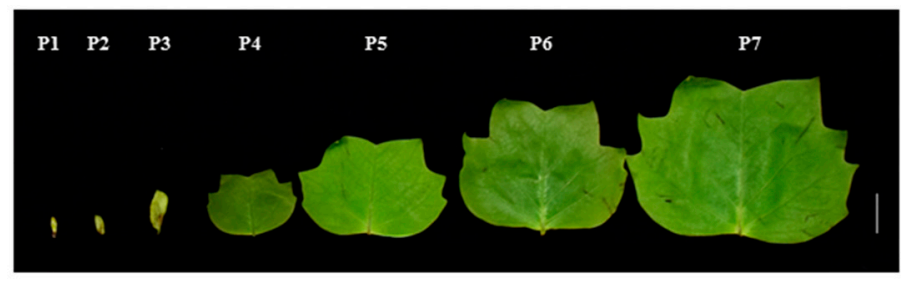

B

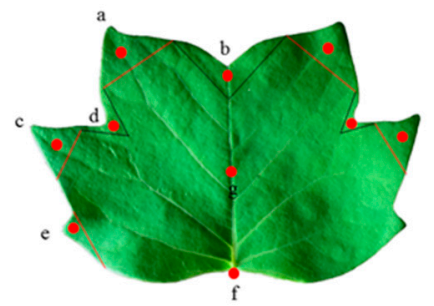

Figure 9. Schematic diagram of sampling in L. tulipifera. (A) Different stages of leaf development in L. tulipifera. P1-P2: leaf bud growth stage; P3: young leaf stage; P4-P6: mature stage; P7: senescence stage; (B) Different parts of leaf in L. tulipifera, a, c, and e: leaf tooth tip, b and d: leaf tooth sinus, g: middle of leaf, f: petiole.

Transgenic and wild-type plants were grown in a Columbia-0 (Col-0) background with Nicotiana benthamiana, and planted in a 6:3:1 mixture of potting soil: vermiculite: perlite in a $23{ }^{\circ} \mathrm{C}$ illumination incubators under long days (16 h light $/ 8 \mathrm{~h}$ dark, $80 \%$ humidity).

\subsection{Rapid Amplification of cDNA Ends (RACE), Sequencing, and Sequence Analysis of LtuJAG}

The JAG EST number (gnl I Liriodendron I b4_c58305) was obtained from the transcriptome database of L. tulipifera (http://jlmwiki.plantbio.uga.edu/aagp/) (accessed on 24 July 2018). RACE cloned primers were designed using Oligo 7 software (Table S1). RACE was conducted using a SMARTer ${ }^{\circledR}$ RACE 5/3 kit (Takara Biomedical Technology, Beijing, China) according to the manufacturer's instructions for amplifying full-length $L t u J A G$, and the amplified PCR product was then ligated into the pEASY vector using a pEASY-Blunt Zero cloning kit (Transgen Biotech, Beijing, China) and verified by sequencing (GenScript Biomedical Technology, Nanjing, China).

LtuJAG Open Reading Frames (ORFs) were predicted using the NCBI ORF finder. The physicochemical characteristics of $L t u J A G$ were analyzed using Expasy Protparam (https: / / web.expasy.org/protparam/) (accessed on 16 October 2021). Multiple alignments of JAG amino acid sequences from various plant species were edited using the DNAMAN 8.0 software. A phylogenetic tree of the aligned sequences was created with MEGA 5.1 software using the neighbor-joining method based on 1000 bootstrap replicates [51]. The promoter was analyzed using the online software PlantCARE to predict putative cis-elements.

\subsection{Subcellular Localization}

To investigate localization where $L t u J A G$ functions in the leaf cell, its subcellular localization was performed using the Agrobacterium injection method as described earlier by Lin et al. [52]. Briefly, the CDS of LtuJAG (without a stop codon) was inserted into the pBI121 vector containing the 35S promoter with an eGFP protein, according to the 
CloneExpress ${ }^{\circledR}$ Ultra One Step Cloning Kit instructions (Vazyme Biotech, Nanjing, China). Next, the constructed 35S::LtuJAG-GFP and the positive control 35S::eGFP plasmids were inserted into Agrobacterium GV3101, which was then injected into the N. benthamiana leaves [53]. To monitor the location of $L t u J A G$ more precisely, the nucleus-specific dye DAPI was utilized after transfection at $22{ }^{\circ} \mathrm{C}$ for $24 \mathrm{~h}$ in the dark. We observed the green fluorescent protein (eGFP) in the tobacco leaves at 2 days after transfection using a Zeiss LSM 710 confocal microscope (Carl Zeiss AG, Jena, Germany). Green fluorescence signals may appear in the cell membrane, nucleus, and cytoplasm. DAPI is a blue fluorescence signal that represents the nucleus location.

\subsection{Agrobacterium-Mediated Transformation}

To generate the LtuJAG-overexpressing transgenic A. thaliana, the LtuJAG coding region was cloned into an improved pBI121-GUS vector downstream of the $35 S$ promoter through the $\mathrm{XbaI}$ and BamHI sites to generate the plasmid 35S::pBI121-LtuJAG. This plasmid was inserted into Agrobacterium GV3101, which was transferred to A. thaliana (Col0 ) using the floral-dip method [54]. The $T_{0}$ seeds of the transgenic plants were screened using a $1 / 2 \mathrm{MS}$ solid medium containing $50 \mathrm{mg} / \mathrm{L}$ kanamycin. The positive seedlings could grow normally about 10 days after seed germination-also observed in transgenic lines of the $T_{1}$ generation. The selection of homozygous transgenic lines through $T_{2}$ and $T_{3}$ generations was performed as described by Mahmood et al. [55]. To monitor the transcript levels of transgene in $L t u J A G$ transgenic lines, total RNA was isolated from the rosette leaves of WT (Col-0) and LtuJAG transgenic plants at 28 days after seed germination using a DP441-RNAprep Pure Plant Kit (Tiangen, Beijing, China) according to the manufacturer's instructions. Primer pairs, LtuJAG-qRT-F and LtuJAG-qRT-R, were used in quantitative RT-PCR. AtACTIN2 was used as an internal control to normalize the expression values of the transgene transcript level [56]. The used primers were listed in Table S1. The relative expression was calculated using the $2^{-\Delta \Delta C t}$ method [57]. Three biological replicates and three technical replicates. Data were analyzed statistically using the one-way ANOVA Tukey's test.

\subsection{GUS Histochemical Assay and Phenotypic Characterization}

It is known that JAG is a class of transcription factor that is specifically expressed in the apical meristem. To investigate $L t u J A G$ promoter expression pattern during differentiation of the apical meristem, we cloned promoter sequences. About $2 \mathrm{~Kb}$ bp fragments upstream of the translational region of the LtuJAG gene were inserted into the plasmid pBI121-GUS at the corresponding restriction sites in place of the $35 \mathrm{~S}$ promoter region, thereby forming a pBI121-pLtuJAG::GUS construct and transformed into A. thaliana. $\mathrm{T}_{2}$ generation transgenic positive lines were used as research material after screening and identification, these seeds and seedlings at the two-leaf, four-leaf, and six-leaf stages were collected, respectively, at 0 , $2,4,6,8,12$ days after seed germination. To understand the tissue-expression pattern, we collected leaves, flowers, stems and siliques from the reproductive stage at 42 days after seed germination, of which GUS activity was detected using GUS histochemical analysis, specific method as previously described by Jefferson et al. [58]. First, tissue samples $(1 \mathrm{~g}$ each) were submerged in $10 \mathrm{~mL} \mathrm{X-gluc} \mathrm{solution} \mathrm{(0.1 \%} \mathrm{5-bromo-4-chloro-3-indolyl-beta-D-}$ glucuronic acid (X-gluc), $1 \%$ dimethyl formamide, $50 \mathrm{mM}$ sodium phosphate [pH 7.0]) at $37^{\circ} \mathrm{C}$ for $24 \mathrm{~h}$. The treated tissues were then decolorized in $75-100 \%$ ethanol. The samples were photographed using a Leica DM500 microscope (Shanghai, China).

To explore the effect of the $L t u J A G$ gene on leaf morphology, the 5-12th rosette leaves (except 1-4th cotyledons) of the $\mathrm{T}_{3}$ generation homozygous WT and LtuJAG overexpression lines grown for 32 days, were photographed using a Fujifilm Finepix S5100 digital camera (Tokyo, Japan), and the JPEG images were used to calculate the lamina length, lamina width, petiole length, lamina area of 12 replicated individual lines using Digimizer (www. digimizer.com) (accessed on 12 November 2021) software, the specific method referring to Luo et al. [59]. We aimed at studying the leaf size differences between the wild-type and 
overexpression strains and therefore data were analyzed statistically using Student's $t$-test to pairwise comparison, and differences were considered significant when values $p<0.01$ and $p<0.05$.

\subsection{Real-Time Quantitative PCR}

To study the temporal expression pattern of genes associated with leaf serration, we examined the transcript levels of leaf serration related genes in wild-type $A$. thaliana leaves at different growth stages and confirmed all genes were specifically expressed at leaf primordia differentiation (10 days after seed germination), and to some extent, some genes were not even expressed at the maturation ( 25 days after seed germination), reproductive (40 days after seed germination), and senescent stages (50 days after seed germination). To explore the effect of $L t u J A G$ on leaf serration related genes, triplicate seedling samples of the homozygous WT and LtuJAG overexpression lines (with the lobed leaf phenotype) at the initial leaf primordia stage (10 days after seed germination), were frozen in liquid nitrogen and stored at $-80{ }^{\circ} \mathrm{C}$ in preparation for gene expression testing using real-time quantitative PCR.

Total RNA was extracted from different samples of $A$. thaliana and L. tulipifera using a total RNA extraction kit (Tiangen, Beijing, China). cDNA was synthesized using the PrimeScript II 1st strand cDNA Synthesis Kit (TaKaRa Biomedical Technology). Quantitative real-time PCR was performed in triplicate using a SYBR Premix Ex Taq kit (TaKaRa Biomedical Technology) on an Applied Biosystems real-time PCR system. AtACTIN2 of A. thaliana and LcActin97 of Liriodendron chinense were used as internal controls with the primers listed in Table S1 [56,60]. For PCR, initial denaturation was performed at $95{ }^{\circ} \mathrm{C}$ for $60 \mathrm{~s}$, which was followed by 40 cycles of $5 \mathrm{~s}$ at $95^{\circ} \mathrm{C}$ and $34 \mathrm{~s}$ at $60{ }^{\circ} \mathrm{C}$ [61]. Three biological replicates and three technical replicates. The relative expression was calculated using the $2^{-\Delta \Delta C t}$ method [56]. Data were analyzed statistically using the one-way ANOVA Tukey's test.

\section{Conclusions}

In this study, we cloned and characterized the LtuJAG gene involved in the development of leaf polarity in L. tulipifera. This gene is a nuclear localized transcription inhibitor that is highly expressed in the lateral organs. We speculate that $L t u J A G$ activates the boundary limiting factors $J L O$ and ATH1 and upregulates the negative regulator of the KNOX I, thereby causing a downregulation of the KNOX I gene. This may disrupt the balance of endogenous hormones, affecting the development of leaf primordia and producing defective leaves. Our study provides evidence that $J A G$ plays a role in the development of leaf polarity.

Supplementary Materials: The following supporting information can be downloaded at: https: / / www.mdpi.com/article/10.3390/ijms23031322/s1.

Author Contributions: L.W. designed and conducted the experiments, and wrote the paper; S.W. participated in conducting the experiments and revised the paper; Z.T. participated in the sample collection, data analysis and paper revision; Y.Z. grew and maintained the plant materials; H.L. conceived the project and revised the paper. All authors have read and agreed to the published version of the manuscript.

Funding: This research was funded by the National Natural Science Foundation of China (grant numbers 31770718 (to H.L. (Huogen Li)) and 31470660 (to H.L. (Huogen Li))) and a project funded by the priority academic program development of Jiangsu higher education institutions (PAPD).

Institutional Review Board Statement: Not applicable.

Informed Consent Statement: Not applicable.

Data Availability Statement: Not applicable. 
Acknowledgments: Arabidopsis thaliana (Col-0) and Nicotiana benthamiana wild type used in this study were kindly provided by Qiang Chen (Nanjing Forestry University, Nanjing, China), we are thankful for his presents.

Conflicts of Interest: The authors declare that they have no conflict of interest.

\section{References}

1. Bowman, J.L.; Eshed, Y.; Baum, S.F. Establishment of polarity in angiosperm lateral organs. Trends Genet. 2002, 18, 134-141. [CrossRef]

2. Du, F.; Guan, C.M.; Jiao, Y.L. Molecular mechanisms of leaf morphogenesis. Mol. Plant 2018, 11, 1117-1134. [CrossRef]

3. Vogel, S. Leaves in the lowest and highest winds: Temperature, force and shape. New Phytol. 2009, 183, 13-26. [CrossRef]

4. Wang, H.F.; Kong, F.J.; Zhou, C.E. From genes to networks: The genetic control of leaf development. J. Integr. Plant Biol. 2021, 63, 1181-1196. [CrossRef]

5. Barton, M.K. Twenty years on: The inner workings of the shoot apical meristem, a developmental dynamo. Dev. Biol. 2010, 341, 95-113. [CrossRef]

6. Long, J.A.; Moan, E.I.; Medford, J.I.; Barton, M.K. A member of the KNOTTED class of homeodomain proteins encoded by the SHOOTMERISTEMLESS gene of Arabidopsis. Nature 1996, 379, 66-69. [CrossRef]

7. Belles-Boix, E.; Hamant, O.; Witiak, S.M.; Morin, H.; Traas, J.; Pautot, V. KNAT6: An Arabidopsis homeobox gene involved in meristem activity and organ separation. Plant Cell 2006, 18, 1900-1907. [CrossRef]

8. Byrne, M.E.; Simorowski, J.; Martienssen, R.A. ASYMMETRIC LEAVES1 reveals KNOX gene redundancy in Arabidopsis. Development 2002, 129, 1957-1965. [CrossRef]

9. Guo, M.J.; Thomas, J.; Collins, G.; Timmermans, M.C.P. Direct repression of KNOX loci by the ASYMMETRIC LEAVES1 complex of Arabidopsis. Plant Cell 2008, 20, 48-58. [CrossRef]

10. Waites, R.; Selvadurai, H.R.; Oliver, I.R.; Hudson, A. The PHANTASTICA gene encodes a MYB transcription factor involved in growth and dorsoventrality of lateral organs in Antirrhinum. Cell 1998, 93, 779-789. [CrossRef]

11. Ha, C.M.; Jun, J.H.; Nam, H.G.; Fletcher, J.C. BLADE-ON-PETIOLE 1 and 2 control Arabidopsis lateral organ fate through regulation of LOB domain and adaxial-abaxial polarity genes. Plant Cell 2007, 19, 1809-1825. [CrossRef] [PubMed]

12. Jun, J.H.; Ha, C.M.; Fletcher, J.C. BLADE-ON-PETIOLE 1 coordinates organ determinacy and axial polarity in Arabidopsis by directly activating ASYMMETRIC LEAVES2. Plant Cell 2010, 22, 62-76. [CrossRef] [PubMed]

13. Khan, M.; Xu, H.S.; Hepworth, S.R. BLADE-ON-PETIOLE genes: Setting boundaries in development and defense. Plant Sci. 2014, 215-216, 157-171. [CrossRef] [PubMed]

14. Furutani, M.; Vernoux, T.; Traas, J.; Kato, T.; Tasaka, M.; Aida, M. PIN-FORMED1 and PINOID regulate boundary formation and cotyledon development in Arabidopsis embryogenesis. Development 2004, 131, 5021-5030. [CrossRef]

15. Kumar, R.; Kushalappa, K.; Godt, D.; Pidkowich, M.S.; Pastorelli, S.; Hepworth, S.R.; Haughn, G.W. The Arabidopsis BEL1-LIKE HOMEODOMAIN proteins SAW1 and SAW2 act redundantly to regulate KNOX expression spatially in leaf margins. Plant Cell 2007, 19, 2719-2735. [CrossRef]

16. Borghi, L.; Bureau, M.; Simon, R. Arabidopsis JAGGED LATERAL ORGANS is expressed in boundaries and coordinates KNOX and PIN activity. Plant Cell 2007, 19, 1795-1808. [CrossRef]

17. Hepworth, S.R.; Zhang, Y.L.; McKim, S.; Li, X.; Haughn, G.W. BLADE-ON-PETIOLE dependent signaling controls leaf and floral patterning in Arabidopsis. Plant Cell 2005, 17, 1434-1448. [CrossRef]

18. Norberg, M.; Holmlund, M.; Nilsson, O. The BLADE-ON-PETIOLE genes act redundantly to control the growth and development of lateral organs. Development 2005, 132, 2203-2213. [CrossRef]

19. David-Schwartz, R.; Koenig, D.; Sinha, N.R. LYRATE is a key regulator of leaflet initiation and lamina outgrowth in Tomato. Plant Cell 2009, 21, v3093-v3104. [CrossRef]

20. Ohno, C.K.; Reddy, G.V.; Heisler, M.G.B.; Meyerowitz, E.M. The Arabidopsis JAGGED gene encodes a zinc finger protein that promotes leaf tissue development. Development 2004, 131, 1111-1122. [CrossRef]

21. Dinneny, J.R.; Yadegari, R.; Fischer, R.L.; Yanofsky, M.F.; Weigel, D. The role of JAGGED in shaping lateral organs. Development 2004, 131, 1101-1110. [CrossRef] [PubMed]

22. Schiessl, K.; Kausika, S.; Southam, P.; Bush, M.; Sablowski, R. JAGGED controls growth anisotropy and coordination between cell size and cell cycle during plant organogenesis. Curr. Biol. 2012, 22, 1739-1746. [CrossRef] [PubMed]

23. Xu, B.; Li, Z.Y.; Zhu, Y.; Wang, H.; Ma, H.; Dong, A.W.; Huang, H. Arabidopsis genes AS1, AS2, and JAG negatively regulate boundary-specifying genes to promote sepal and petal development. Plant Physiol. 2008, 146, 566-575. [CrossRef] [PubMed]

24. Sperschneider, J.; Catanzariti, A.M.; DeBoer, K.; Petre, B.; Gardiner, D.M.; Singh, K.B.; Dodds, P.N.; Taylor, J.M. LOCALIZER: Subcellular localization prediction of both plant and effector proteins in the plant cell. Sci. Rep. 2017, 7, 44598. [CrossRef]

25. Jeong, N.; Suh, S.J.; Kim, M.H.; Lee, S.; Moon, J.K.; Kim, H.S.; Jeong, S.C. LN is a key regulator of leaflet shape and number of seeds per pod in soybean. Plant Cell 2012, 24, 4807-4818. [CrossRef] [PubMed]

26. Lim, C.; Baek, W.; Jung, J.; Kim, J.; Lee, S. Function of $A B A$ in stomatal defense against biotic and drought stresses. Int. J. Mol. Sci. 2015, 16, 15251-15270. [CrossRef] [PubMed]

27. Grant, M.; Lamb, C. Systemic immunity. Curr. Opin. Plant Biol. 2006, 9, 414-420. [CrossRef] 
28. Von Dahl, C.C.; Winz, R.A.; Halitschke, R.; Kuhnemann, F.; Gase, K.; Baldwin, I.T. Tuning the herbivore-induced ethylene burst: The role of transcript accumulation and ethylene perception in Nicotiana attenuate. Plant J. 2007, 51, 293-307. [CrossRef]

29. Tiwari, P.; Indoliya, Y.; Chauhan, A.S.; Singh, P.; Singh, P.K.; Singh, P.C.; Srivastava, S.; Pande, V.; Chakrabarty, D. Auxin-salicylic acid cross-talk ameliorates OsMYB-R1 mediated defense towards heavy metal, drought and fungal stress. J. Hazard. Mater. 2020, 399, 122811. [CrossRef]

30. Wei, Z.P.; Ye, J.F.; Zhou, Z.Q.; Chen, G.; Meng, F.J.; Liu, Y.F. Isolation and characterization of ProWRKY, an abiotic stress-related WRKY transcription factor from Polygonatum odoratum. Physiol. Mol. Biol. Plants 2021, 27, 1-9. [CrossRef]

31. Horigome, A.; Nagasawa, N.; Ikeda, K.; Ito, M.; Itoh, J.; Nagato, Y. Rice open beak is a negative regulator of class 1 KNOX genes and a positive regulator of class B floral homeotic gene. Plant J. 2009, 58, 724-736. [CrossRef] [PubMed]

32. Xiao, H.; Tang, J.F.; Li, Y.F.; Wang, W.M.; Li, X.B.; Jin, L.; Xie, R.; Luo, H.F.; Zhao, X.F.; Meng, Z.; et al. Stamenless 1, encoding a single $\mathrm{C}_{2} \mathrm{H}_{2}$ zinc finger protein, regulates floral organ identity in rice. Plant J. 2009, 59, 789-801. [CrossRef] [PubMed]

33. Duan, Y.L.; Li, S.P.; Chen, Z.W.; Zheng, L.L.; Diao, Z.J.; Zhou, Y.C.; Lan, T.; Guan, H.Z.; Pan, R.S.; Xue, Y.B.; et al. Dwarf and deformed flower1, encoding an F-box protein, is critical for vegetative and floral development in rice (Oryza sativa L.). Plant J. 2012, 72, 829-842. [CrossRef] [PubMed]

34. Li, Y.J. The Preliminary Investigation of Arabidopsis Transcription Factor JAGGED in Regulating the Morphogenesis of Petal Conical Cells. Ph.D. Thesis, Fujian Agriculture and Forestry University, Fuzhou, China, 2018.

35. Zhang, G.H.; Xu, Q.; Zhu, X.D.; Qian, Q.; Xue, H.W. SHALLOT-LIKE1 is a KANADI transcription factor that modulates rice leaf rolling by regulating leaf abaxial cell development. Plant Cell 2009, 21, 719-735. [CrossRef]

36. Wang, H.; Zhou, Y.M.; Gilmer, S.; Whitwill, S.; Fowke, L.C. Expression of the plant cyclin dependent kinase inhibitor ICK1 affects cell division, plant growth and morphology. Plant J. 2000, 25, 613-623. [CrossRef]

37. Cole, M.; Nolte, C.; Werr, W. Nuclear import of the transcription factor SHOOT MERISTEMLESS depends on heterodimerization with BLH proteins expressed in discrete sub-domains of the shoot apical meristem of Arabidopsis thaliana. Nucleic Acids Res. 2006, 34, 1281-1292. [CrossRef] [PubMed]

38. Spinelli, S.V.; Martin, A.P.; Viola, I.L.; Gonzalez, D.H.; Palatnik, J.F. A mechanistic link between STM and CUC1 during Arabidopsis development. Plant Physiol. 2011, 156, 1894-1904. [CrossRef]

39. Nath, U.; Crawford, B.C.; Carpenter, R.; Coen, E. Genetic control of surface curvature. Science 2003, 299, 1404-1407. [CrossRef] [PubMed]

40. Ori, N.; Cohen, A.R.; Etzioni, A.; Brand, A.; Yanai, O.; Shleizer, S.; Menda, N.; Amsellem, Z.; Efroni, I.; Pekker, I.; et al. Regulation of LANCEOLATE by miR319 is required for compound leaf development in tomato. Nat. Genet. 2007, 39, 787-791. [CrossRef]

41. Nelissen, H.; Rymen, B.; Jikumaru, Y.; Demuynck, K.; van Lijsebettens, M.; Kamiya, Y.; Inze, D.; Beemster, G.T. A local maximum in gibberellin levels regulates maize leaf growth by spatial control of cell division. Curr. Biol. 2012, 22, 1183-1187. [CrossRef]

42. Rubio-Somoza, I.; Zhou, C.M.; Confraria, A.; Martinho, C.; von Born, P.; Baena-Gonzalez, E.; Wang, J.W.; Weigel, D. Temporal control of leaf complexity by miRNA-regulated licensing of protein complexes. Curr. Biol. 2014, 24, 2714-2719. [CrossRef] [PubMed]

43. Huang, S.; Raman, A.S.; Ream, J.E.; Fujiwara, H.; Cerny, R.E.; Brown, S.M. Overexpression of 20-oxidase confers a gibberellinoverproduction phenotype in Arabidopsis. Plant Physiol. 1998, 118, 773-781. [CrossRef] [PubMed]

44. Achard, P.; Gusti, A.; Cheminant, S.; Alioua, M.; Dhondt, S.; Coppens, F.; Beemster, G.T.; Genschik, P. Gibberellin signaling controls cell proliferation rate in Arabidopsis. Curr. Biol. 2009, 19, 1188-1193. [CrossRef] [PubMed]

45. Bilsborough, G.D.; Runions, A.; Barkoulas, M.; Jenkins, H.W.; Hasson, A.; Galinha, C.; Laufs, P.; Hay, A.; Prusinkiewicz, P.; Tsiantis, M. Model for the regulation of Arabidopsis thaliana leaf margin development. Proc. Natl. Acad. Sci. USA 2011, 108, 3424-3429. [CrossRef] [PubMed]

46. Tameshige, T.; Okamoto, S.; Lee, J.S.; Aida, M.; Tasaka, M.; Torii, K.U.; Uchida, N. A secreted peptide and its receptors shape the auxin response pattern and leaf margin morphogenesis. Curr. Biol. 2016, 26, 2478-2485. [CrossRef] [PubMed]

47. Jasinski, S.; Piazza, P.; Craft, J.; Hay, A.; Woolley, L.; Rieu, I.; Phillips, A.; Hedden, P.; Tsiantis, M. KNOX action in Arabidopsis is mediated by coordinate regulation of cytokinin and gibberellin activities. Curr. Biol. 2005, 15, 1560-1565. [CrossRef] [PubMed]

48. Sakamoto, T.; Kamiya, N.; Ueguchi-Tanaka, M.; Iwahori, S.; Matsuoka, M. KNOX homeodomain protein directly suppresses the expression of a gibberellin biosynthetic gene in the tobacco shoot apical meristem. Genes Dev. 2001, 15, 581-590. [CrossRef] [PubMed]

49. Ma, J.K.; Wei, L.M.; Li, J.Y.; Li, H.G. The analysis of genes and phytohormone metabolic pathways associated with leaf shape development in Liriodendron chinense via De Novo transcriptome sequencing. Genes 2018, 9, 577. [CrossRef]

50. Li, K.; Chen, L.; Feng, Y.; Yao, J.; Li, B.; Xu, M.; Li, H. High genetic diversity but limited gene flow among remnant and fragmented natural populations of Liriodendron chinense. Biochem. Syst. Ecol. 2014, 54, 230-236. [CrossRef]

51. Saitou, N.; Nei, M. The neighbor-joining method: A new method for reconstructing phylogenetic trees. Mol. Biol. Evol. 1987, $4,406-425$.

52. Lin, Y.; Hou, H.; Zhang, Y.; Hou, X. Overexpression of a pak choi gene, BcAS2, causes leaf curvature in Arabidopsis thaliana. Genes 2021, 12, 102. [CrossRef] [PubMed]

53. Meng, C.; Sui, N. Overexpression of maize MYB-IF35 increases chilling tolerance in Arabidopsis. Plant Physiol. Biochem. 2019, 135, 167-173. [CrossRef] [PubMed]

54. Clough, S.J.; Bent, A.F.J. Floral dip: A simplified method for Agrobacterium-mediated transformation of Arabidopsis thaliana. Plant J. 2010, 16, 735-743. [CrossRef] [PubMed] 
55. Mahmood, K.; El-Kereamy, A.; Kim, S.H.; Nambara, E.; Rothstein, S.J. ANAC032 positively regulates age-dependent and stress-induced senescence in Arabidopsis thaliana. Plant Cell Physiol. 2016, 57, 2029-2046. [CrossRef] [PubMed]

56. Wan, X.; Peng, L.; Xiong, J.; Li, X.; Wang, J.; Li, X.; Yang, Y. AtSIBP1, a novel BTB domain-containing protein, positively regulates salt signaling in Arabidopsis thaliana. Plants 2019, 8, 573. [CrossRef] [PubMed]

57. Livak, K.J.; Schmittgen, T.D. Analysis of relative gene expression data using real-time quantitative PCR and the 2(T) (-Delta Delta C) method. Methods 2001, 25, 402-408. [CrossRef] [PubMed]

58. Jefferson, R.A.; Kavanagh, T.A.; Bevan, M.W. GUS fusions: Beta-glucuronidase as a sensitive and versatile gene fusion marker in higher plants. EMBO J. 1987, 13, 3901-3907. [CrossRef]

59. Luo, M.; Yu, C.W.; Chen, F.F.; Zhao, L.M.; Tian, G.; Liu, X.C.; Cui, Y.H.; Yang, J.Y.; Wu, K.Q. Histone deacetylase HDA6 is functionally associated with AS1 in repression of KNOX genes in Arabidopsis. PLoS Genet. 2012, 8, e1003114. [CrossRef]

60. Tu, Z.; Hao, Z.; Zhong, W.; Li, H. Identification of suitable reference genes for RT-qPCR assays in Liriodendron chinense (Hemsl.). Forests 2019, 10, 441. [CrossRef]

61. Bustin, S.A.; Benes, V.; Garson, J.A.; Hellemans, J.; Huggett, J.; Kubista, M.; Mueller, R.; Nolan, T.; Pfaffl, M.W.; Shipley, G.L. The MIQE guidelines: Minimum information for publication of quantitative real-time PCR experiments. Clin. Chem. 2009, 55, 611-622. [CrossRef] 